\title{
Rheology of Cementitious Materials: Alkali-Activated Materials or Geopolymers
}

\author{
F. Puertas ${ }^{1}$, M.M: Alonso ${ }^{1}$, S. Gismera ${ }^{1}$, M. Lanzón ${ }^{2}$ and M.T. Blanco-Varela ${ }^{1}$ \\ ${ }^{1}$ Cements andRecycling Materials Dpt. Eduardo Torroja Institute for Construction Sciences (IETcc-CSIC), Madrid, Spain \\ ${ }^{2}$ Arquitectura y Tecnología de la Edificación Dpt. Universidad Politécnica de Cartagena, Cartagena (Murcia), Spain
}

\begin{abstract}
A clear alternative to reach the goal of sustainable development in the Construction Sector is the development of alternative building materials to Ordinary Portland Cement (OPC) in a more energetically as well as environmentally eco-efficient way. Alkaline cements (Alkali-Activated Materials, AAMs) and geopolymers meet these requirements; and they are based on the alkali activation of aluminosilicates (mainly waste and industrial by-products, such as blast furnace slag, fly ash and ceramic waste) in highly alkaline solutions. AAMs cements and concretes are notable for being very durable and mechanically resistant. However, to date their rheological behaviour is not well controlled and there is little understanding of it, with very disparate experimental data. Despite this, their rheological behaviour is not fully understood and little is known on the disparate data obtained in AAM pastes. Moreover, the common additives used in the preparation of OPC concretes and the rheology modifiers/controllers are also unstable in the AAMs systems.

Understanding and controlling the rheology of the AAMs systems will ultimately determine whether they can be implemented in the market, and will open up greater competitive possibilities in a crisis-affected sector. A systematic study of the factors that affect the rheological properties of AAMs (pastes, mortars and concretes) is therefore necessary in order to ultimately develop more resistant and durable materials.
\end{abstract}

\section{Introduction}

Rheology is the study of the fluidity and deformation of matter. An understanding of the rheological properties of cement pastes, mortars and concretes is essential to ascertaining their consistency and workability and consequently their ease of casting or placement. In addition, the rheological properties of cement-based materials have a strong influence on their microstructure, mechanical features and durability [1].

A number of methods are in place for determining the fluidity of binders, including slump tests, the compactness factor and the Vebe consistometer. The advantages of these primarily empirical procedures are their low cost, on-site applicability and simplicity. These measurements, may lead, however, to erroneous conclusions, such as assuming that the same slump value indicates similarity between two pastes that may, in fact, be different. Another drawback of these methods is their high sensitivity to the equipments, technicians and test preparations. As a consequence of this, appropriate methods are needed to characterise, with sufficient accuracy, the rheological behaviour of these materials. Cement system fluidity can be determined from physical parameters, such as viscosity or yield stress measured with viscometers or rheometers. Many authors have studied Portland cement paste rheology as a preliminary to determining the rheology of the respective mortars and concretes [2-8].

Rheological testing on Portland cement pastes, normally conducted with viscometers, consists of subjecting the pastes to shear at a given rate to break down the flocs formed mainly due to the hydration of cement grains. The shear rate (rotor speed) is then gradually lowered. As a rule, the down ramp on the shear rate vs shear stress $(\tau)$ curve is a straight line that fits the Bingham model (Equation 1): i.e., where the slope is the plastic viscosity $(\mu)$ and the yintercept the yield stress $\left(\tau_{\mathrm{o}}\right)$. Plastic viscosity is related to the number and size of the flocs, while the yield stress is a measure of the strength and number of inter-floc interrelations that are broken down when shear is applied [4].

$\tau=\tau_{0}+\mu \gamma$

The literature [1] nonetheless describes other models for cement paste rheological behaviour, such as the Ostwald de Waele and Herschel-Bulkley models (Equations 2 and 3, respectively).

Ostwald de Waele model $\tau=K \gamma^{n}$

Herschel-Bulkley model $\tau=\tau_{0}+K \gamma^{n}$ 
where $\mathrm{K}$ is the consistency coefficient $\left(\mathrm{Pa} \cdot \mathrm{s}^{\mathrm{n}}\right)$ and $\mathrm{n}$ is the dimensionless fluidity index.

There are many studies that relate the rheology of OPC concrete with their final performance and behaviour [9]. But very few studies have been conducted on alkaline activated cement paste, mortar or concrete rheology, however.

Alkaline cements (Alkali-Activated Materials, AAMs) and geopolymers cover a wide variety of materials whose differences depend on the origin and composition of the starting aluminosilicates. Alkaliactivated slag and fly ash cements are among the most widely known for their availability and high performance. Due to chemical differences in the starting materials (vitreous blast furnace slag and silicoaluminate fly ash) the reaction products and the microstructure of these cements vary considerably. These features determine the properties of the resulting cements and concretes, which have been shown to exhibit suitable strength development and good durability [10-12]. Those properties, along with drying shrinkage, have likewise been proven to depend heavily on the nature of the activating solution [13, 14].

In general terms, the AAMs are characterized by a good mechanical strength and durability against different aggressive agents [12, 15-20]. However, to date little is known on the physical behaviour of AAMs pastes as well as the intrinsic variability observed in rheological tests. What is more, numerous additives and rheology modifiers widely used in OPC pastes are either unstable or show poor performance in AAMs systems.

In the following section, a brief description of important rheological aspects of AAMs systems (pastes, mortars and concretes) will be analysed. Likewise, the best conditions of dosage and preparation of these materials will be described.

\section{Rheology of AAMs paste, mortar and concretes}

In alkali-activated slag (AAS) cements, the nature of the activator is a key factor in the rheological behaviour of the paste [21]. When the activators are $\mathrm{NaOH}(\mathrm{N})$ or $\mathrm{NaOH} / \mathrm{Na} 2 \mathrm{CO} 3$ (NC) solutions the exhibited consistently (or shear stress) is lower than OPC pastes ones. But when the activator is a waterglass (Wg) solution (sodium silicate hydrate dissolution), the rheology of the pastes is related to the $\mathrm{SiO} 2 / \mathrm{Na} 2 \mathrm{O}$ ratio and $\mathrm{Na} 2 \mathrm{O}$ concentration. Probably, a primary C-S-H gel is formed in this case due to the interaction between silicate ions and $\mathrm{Ca} 2+$ ions from the activator and the slag, respectively. This reaction might explain the fast set of alkali-activated pastes prepared with waterglass. In Figure 1, the variation of shear stress versus time shows the different behaviour observed in OPC and AAS pastes.

Considering the above mentioned facts, longer mixing times could be beneficial (or even necessary) when preparing handling Wg-ASS mixtures.

The rheology of alkali-activated fly ashes (AAFA) pastes depends on temperature; at room temperature the rheology of these pastes is quite similar to those of OPC.
An increase of the concentration of alkaline activator leads to an increase of the viscosity of the pastes.

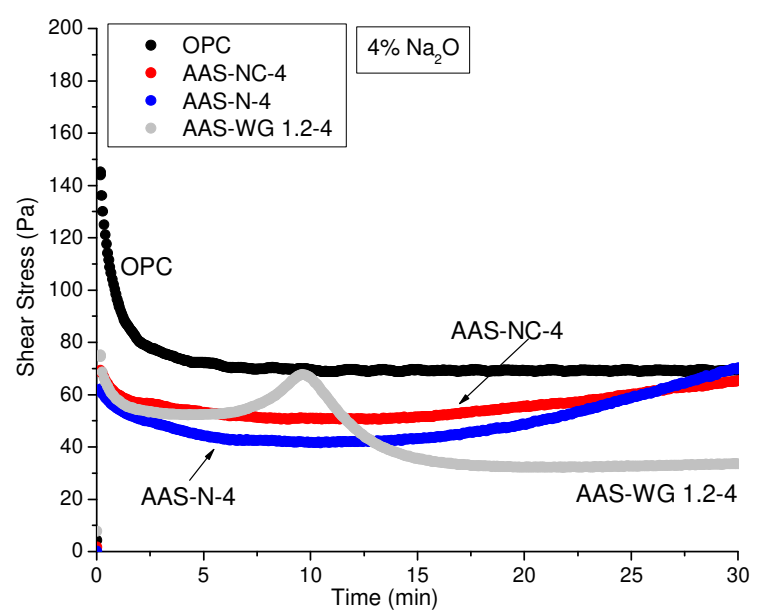

Fig. 1. Shear stress versus time in AAS pastes tested at a constant shear rate of $100 \mathrm{~s}-1$ : effect of the nature of the activator at constant concentration ( $4 \% \mathrm{Na} 2 \mathrm{O}$ by slag mass)

According to different studies, the workability of AAS and AAFA could be more sensitive than that observed in OPC mortars. The effect is more evident when the Liquid/Solid ratio or the amount of aggregate is modified in AAS and AAFA mixtures. Moreover, workability is much more dependent upon the nature of the activator in AAS than in AAFA mortars [22].

OPC, AAS and AAFA mortar rheology was shown to be comparable, although certain considerations around AAMs, such as precursor and activator nature, must be borne in mind. The presence of waterglass ( $\mathrm{Si}$ ) in the activator played a significant role in the rheology of AAS and AAFA mortars, which should be taken into consideration when alkaline-activated materials are casted in place.

At a dry consistency, OPC mortars were found to be more workable than AAS and AAFA mortars at the same L/S ratio. In contrast, after reaching plasticity, AAS and AAFA mortars activated with silicon-containing solutions had greater workability than the respective OPC mortars. In other words, the fluidising effect of silicon was observed in plastic mortars, but not in dry consistency mortars. Under the latter conditions, factors such as particle packing and the physical properties of the precursors and activators (viscosity and surface tension, for instance) appeared to prevail.

Previous studies on pastes and mortars demonstrate the positive effect of mixing time on the rheological behaviour of AAS systems. The slump and rheological behaviour of OPC concrete and AAS concrete are different, especially when waterglass was the alkaline activator. As in pastes and mortars the nature of alkaline activator is the main factor in the rheological behaviour of AAS concretes. Longer mixing protocol has a negative effect on rheological parameters (e.g. dynamic and static yield stress) of OPC and NaOH-AAS concretes. That mixing protocol may produce irreversible coagulation among cement particles that lead to a lower workability. In contrast, in Waterglas-AAS concrete, longer mixing 
times is beneficial to obtain workable mixtures. In this case, longer mixing times would be required to break down flocs within the microstructure, thus improving their rheological behaviour of the pastes and concretes [23].

\section{Final remarks}

The rheology of AAMs is quite different to OPC ones, and it is strongly influenced by the particular nature of the silicoaluminate which is used as precursor. When the precursor is a ground vitreous blast furnace slag (AAS), the nature of the alkaline activator is the key factor in the workability of the system. If waterglass is used as activator, additional mixing time is required to obtain adequate slump and workability. No changes are needed when the activators are $\mathrm{NaOH}$ or $\mathrm{NaOH} / \mathrm{Na} 2 \mathrm{CO} 3$ solutions. Finally, the rheology of pastes, mortars and concretes prepared with siliceous fly ash as precursor, is comparable to that observed in OPC systems.

\section{Acknowledgements}

The authors thank the Spanish Ministry of Economy, Industry and Competitiveness for funding the Project BIA2013-47876-C2-1-P.

\section{References}

1. G.H. Tattersall, P.F.G. Banfill The reology of fresh concrete. Ed, Pitman, Advanced Publishing Program (1983).

2. J. Miranda, V. Flores-Alés, J. Barrios, J. Mater. Construcc. 50, 257, 47-55 (2000).

3. C.F.Ferraris, K.H. Obla, R. Hill, R. Cem Concr. Res. 31, 245-255 (2001).

4. P.F.G. Banfill, Proc. XI ICCC (Durban, South Africa), pp. 50-62 (2003).

5. J. Golaszewski, J. Cement Wapno Beton. 1, 17-28 (2006)

6. O. Burgos-Montes, M. Palacios, P. Rivilla, F. Puertas, F. Constr Build Mater 31, 300-309 (2012).

7. M.M: Alonso. M. Palacios, F. Puertas, A.G. de la Torre, M.A.G. Aranda Mater Construcc. 57 286: 65$81(2007$

8. M.M. Alonso, M. Palacios, F. Puertas Cem. Concr. Comp, 35 151-162 (2013).

9. K. Kovler, N. Roussel, N. Cem Concr Res 41, 775792 (2011).

10. C. Shi, A. Fernández-Jiménez, A. Palomo Cem. Concr. Res., 41 (7), 750-763 (2011).

11. C. Duran Atiş, C. Bilim, Ö. Çelik, O. Karahan. Constr.Build. Mater 23 (1) 548-555 (2009).

12. A. Fernández-Jiménez, J.G. Palomo, F. Puertas, Cem. Concr. Res., 29 (8), 1313-1321 (1999).

13. M. Palacios, F. Puertas Cem. Concr. Res. 37 (5) 691702 (2007).

14. F. Puertas, M. Palacios, T. Vázquez J. Mater. Sci., 41, 3071-3082 (2006).
15. A. Favier, G. Habert, J.B.D'Éspinose De Lacaillerie, N. Roussel Cem. Concr. Res., 48, 9-16 (2013).

16. F. Pacheco-Torgal, J.A. Labrincha, C. Leonelli, A. Palomo, P. Chindaprasirt Handbook of Alkaliactivated cements, mortars and concretes, Woodhead Publishing (2015).

17. C. Shi, P. Krivenko, D. Roy. Alkali-Activated Cements and Concretes, Taylor and Francis, London and New York. (2006).

18. J.L. Provis, A. Palomo, C. Shi Cem. Concr. Res. 78 110-125(2015)

19. J.L. Provis, Mater. Struct. 47, 11-25 (2014)

20. J.L. Provis, S.J. Van Deventer. Alkali-Activated Materials. State of Art Report, RILEM TC 224AAM. Ed. Springer (2014).

21. F. Puertas, C. Varga, M.M. Alonso Cem Concr Com, 53, 279-288 (2014).

22. M.M. Alonso, S. Gismera, M. T. Blanco, M. Lanzón, F. Puertas. Construc Build Mat, 145, 576-587 (2017)

23. F. Puertas, M.M. Alonso, M. Torres-Carrasco, B. González-Fonteboa, I. González-Taboada, G. Rojo, F. Martínez-Abella. $10^{\text {th }}$ ACI/RILEM Int Conf Cement Mat and Alternative Binders for Sustainable Concrete. Montreal (2017) 